\title{
Sub-brow blepharoplasty for simultaneous correction of dermatochalasis with upper eyelid hooding and sunken eyelids
}

Jin Bin Kim¹, Jong Chan Kim², Han Byeol Jin ${ }^{1}$, Sun Ok Kim², Jee Hyeok Chung ${ }^{1}$

${ }^{1}$ Department of Plastic and Reconstructive Surgery, Myongji Hospital, Goyang; ${ }^{2}$ Tami Plastic Surgery, Incheon, Korea
Periorbital dermatochalasis with upper eyelid hooding, brow ptosis, and sunken eyelids may appear with age. Because classic blepharoplasty is unable to correct all these issues, we developed a single operation, which we present herein, to correct dermatochalasis accompanied by sunken eyelids. This sub-brow approach is used with simultaneous browpexy by fixing the orbital portion of the orbicularis oculi muscle (OOM) to the periosteum immediately above the supraorbital rim using sutures with 3 or 4 points of fixation and correcting sunken eyelids by burying the elevated dermis, fat, and OOM after de-epithelization in the lower flap of the sunken upper eyelid along the submuscular plane. This method enables the correction of sunken eyelids during the same operation without requiring an additional procedure, and offers the advantages of a shortened operation time and decreased cost. The presence of sunken eyelids in patients with dermatochalasis and severe lateral hooding may be corrected by the procedure described herein, thereby achieving periorbital rejuvenation while maintaining the original shape of the eyes.

Keywords Blepharoplasty / Eyelids / Skin aging / Rejuvenation / Middle aged

\section{INTRODUCTION}

With aging, characteristic changes occur in the periorbital area, including dermatochalasis with lateral hooding, a low resting brow (brow ptosis), and sunken eyelids; these changes can occur together or individually, and they can cause visual field obstruction, maceration of the folded eyelid skin, and an old and tired appearance.

Upper blepharoplasty is commonly performed to correct agingrelated changes. However, classic blepharoplasty involves excising the redundant upper eyelid skin above the double fold line and

Received: Sep 12, 2018 Revised: Sep 17, 2018 Accepted: Sep 21, 2018 Correspondence: Jee Hyeok Chung Department of Plastic and Reconstructive Surgery, Myongji Hospital, 55 Hwasu-ro 14beon-gil, Deokyang-gu, Goyang 10475, Korea

Tel: +82-31-810-6830, Fax: +82-31-810-6837, E-mail: gabriel@mjh.or.kr

Copyright () 2019 The Korean Society for Aesthetic Plastic Surgery.

This is an Open Access article distributed under the terms of the Creative Commons At tribution Non-Commercial License (http://creativecommons.org/licenses/by-nc/4.0/) which permits unrestricted non-commercial use, distribution, and reproduction in any medium, provided the original work is properly cited. $\quad w w w . e-a a p s . o r g$ pulling the thicker upper skin and suturing it to the double-fold line. This can result in an unnatural-looking double-fold line formed by heavy and thick skin during eye opening. Moreover, the excision of a large amount of redundant upper eyelid skin due to severe hooding can leave a lengthy scar that extends beyond the lateral canthus, or dog-ear formation may occur in the very end portion. In such cases, blepharoplasty using a sub-brow excision is recommended. Even if dermatochalasis is corrected using blepharoplasty alone, a postoperative brow drop may persist due to undetected compensated brow ptosis, which may worsen the individual's aged and tired appearance. Moreover, if redundant skin and periorbital fat are excised surgically, sunken eyelids can become more visible, because such a procedure causes the eyelids to be flattened against the orbital septum, which may require additional corrective treatment [1] such as micro-fat grafting [2-4], dermofat grafting, fasciafat grafting [5], or the use of alloplastic materials [6].

Accordingly, we introduce sub-brow blepharoplasty (SBB) to resolve dermatochalasis accompanied by sunken eyelids in a single operation with simultaneous browpexy and sunken eyelid correction. The orbital portion of the orbicularis oculi muscle (OOM) is 
fixed to the periosteum immediately above the supraorbital rim using sutures with 3 or 4 points of fixation, and sunken eyelids are corrected by burying the elevated dermis, fat, and OOM after deepithelization under the lower flap of the upper sunken eyelid along the submuscular plane.

\section{CASE REPORTS}

From March 2011 to June 2018, the following SBB was performed for the ideal patient among the patients who visited for upper blepharoplasty, and the follow-up duration ranged from 6 months to 4 years (mean, 21 months). Written informed consents were obtained.

The ideal patients for our method are those with relatively high eyebrows and wide eyelids, as well as with upper eyelid dermatochalasis with lateral hooding who have tattooed eyebrows or want to change their eyebrow contour. It is preferable if the patient has preexisting supratarsal folds, was satisfied with the shape of their eyes at a younger age and wants to return to that state, or has sunken eyelids.

This method is also suitable for patients who wish to avoid a lengthy postoperative incisional scar that extends beyond the lateral canthus; thus, it was performed on patients who satisfied these conditions.

Preoperative marking was performed with the patient in the sitting position. A reference line was drawn on the midline of the glabella, after which the supraorbital nerve (SON) and supratrochlear nerve (STN) points were marked laterally at 2.5 and $1.5 \mathrm{~cm}$, respectively. The excised skin was spindle-shaped; if no change to the eyebrow contour was planned, the upper incision line was drawn along the inferior margin of the eyebrow. The amount of skin excision was determined after pinching with forceps, based on which the lower incision line was drawn. The incision did not extend beyond the medial margin of the brow. The amount of excised skin varied, with a length of 40 to $50 \mathrm{~mm}$ and a width of 8 to $12 \mathrm{~mm}$. Local anesthesia was administered using 1:100,000 epinephrine in $2 \%$ lidocaine. Patient anxiety was managed by short-term sedation with intravenous midazolam and ketamine.

To minimize damage to the hair follicles, the upper incision was performed obliquely in a medial-to-lateral direction parallel to the follicles, while the muscle incision was performed in a lateral-tomedial direction. En bloc excision including some skin, subcutaneous fat, and OOM was performed. Then, the OOM at the lateral end of the supraorbital rim, lateral side of the STN, and between the SON and STN was vertically split using Metzenbaum scissors to form a window, and a limited dissection approximately $1 \mathrm{~cm}$ long was performed along the supraperiosteal plane.

The OOM in the upper flap was fixed to the periosteum at the exposed supraorbital rim through the premade windows using 5-0 nylon for 3 points. The fixation locations varied according to the severity of brow ptosis, but it was possible to fix the eyebrow up to a maximum of $8 \mathrm{~mm}$. When necessary, an additional window was added to the medial aspect of the STN to enable 4-point suture fixation. In the more recent cases in this series, the muscle is fixed to the periosteum immediately after muscle splitting, with minimal dissection.

If sunken eyes were noticeable after the browpexy, the tissue excised in the previous step was de-epithelialized and buried along the submuscular plane of the lower flap of the upper eyelid, followed by subcutaneous and skin sutures (Fig. 1). In cases of severely sunken eyelids, the upper eyelid skin was simply de-epithelialized and the OOM based on the upper flap was partially excised and elevated, and buried under the lower flap to increase the engraftment rate. A drain was not inserted; rather, gentle compressive dressings were applied for 3 days and the sutures were removed at 5 to 7 postoperative days.

\section{DISCUSSION}

Thinned skin, weakening of support structures, reduction of fat and bone density, downward movement of surrounding tissues
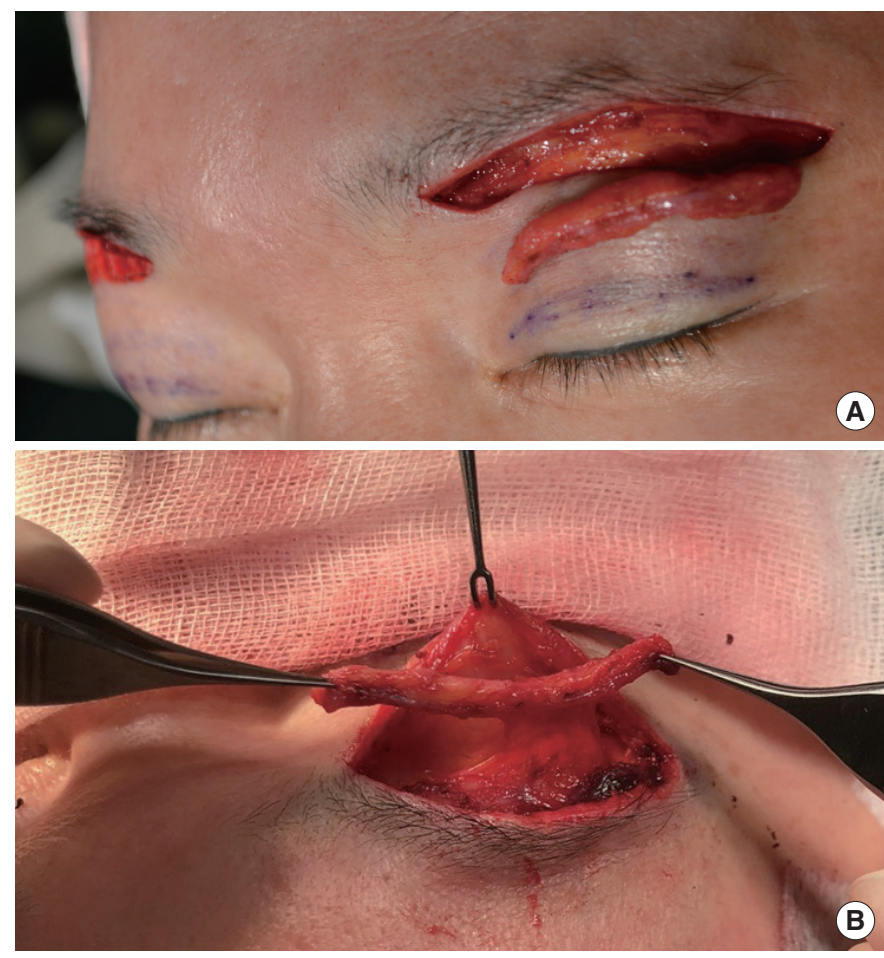

Fig. 1. Details of procedures. (A) After excision of redundant skin, fat, and the orbicularis oculi muscle, de-epithelization was performed. (B) In cases of severely sunken eyelids, we performed a modification for correction of sunken eyelids. After de-epithelization of the redundant skin only, the muscle just below the removed skin was elevated with the middle part attached to the upper flap to maintain the blood supply. 
due to stretching of the forehead and brow, and dermatochalasis due to skin laxity, brow ptosis, and sunken eyelids may occur in the periorbital area with age. Because there is no frontalis muscle at the lateral forehead, lateral hooding of the upper eyelid may be caused by the absence of an upward vector in conjunction with other factors such as skin laxity, actions of the lateral fibers of the OOM and transverse head of the corrugator supercilii, a thick upper eyelid with retro-orbicularis oculi fat (ROOF), and brow ptosis. Asians generally have higher eyebrows, wider upper eyelids [7], and more pretarsal fat and ROOF; as a result, the eyelids project inferiorly, giving the eyes a puffy appearance [8,9]. In contrast, Westerners have more prominent supraorbital rims, relatively lower eyebrows, less postseptal fat, and thinner upper eyelid skin. Thus, Asians are susceptible to skin drooping above the lateral eyelid. However, conventional upper blepharoplasty for skin hooding can leave a long scar and the correction of brow ptosis may be difficult, leading to unintended changes to the eye contour. Therefore, SBB is more suitable since it accomplishes the following [10]: (1) correction of lateral hooding and slanting of the upper eyelids; (2) revision of eyebrow contours and tattoos; (3) easy concealment of initial scars; (4) avoidance of a lengthy lateral scar and dog-ear formation, both of which can occur with classic blepharoplasty; (5) thinning of ROOF and internal brow suspension; (6) medial brow elevation through more direct resection of the brow depressors; and (7) natural-appearing eyelid creases and contours.

Since its introduction in 1976 [11], SBB has become popular in Asian countries, including Japan and Korea $[12,13]$. Previous methods included only removal of the skin layer [10] or en bloc excision of the skin and OOM [12], followed by pulling the lowerflap OOM to the upper-flap OOM. In 2013, a method of en bloc excision of skin and OOM followed by fixation of the lower-flap OOM to the periosteum of the upper flap was introduced [14].
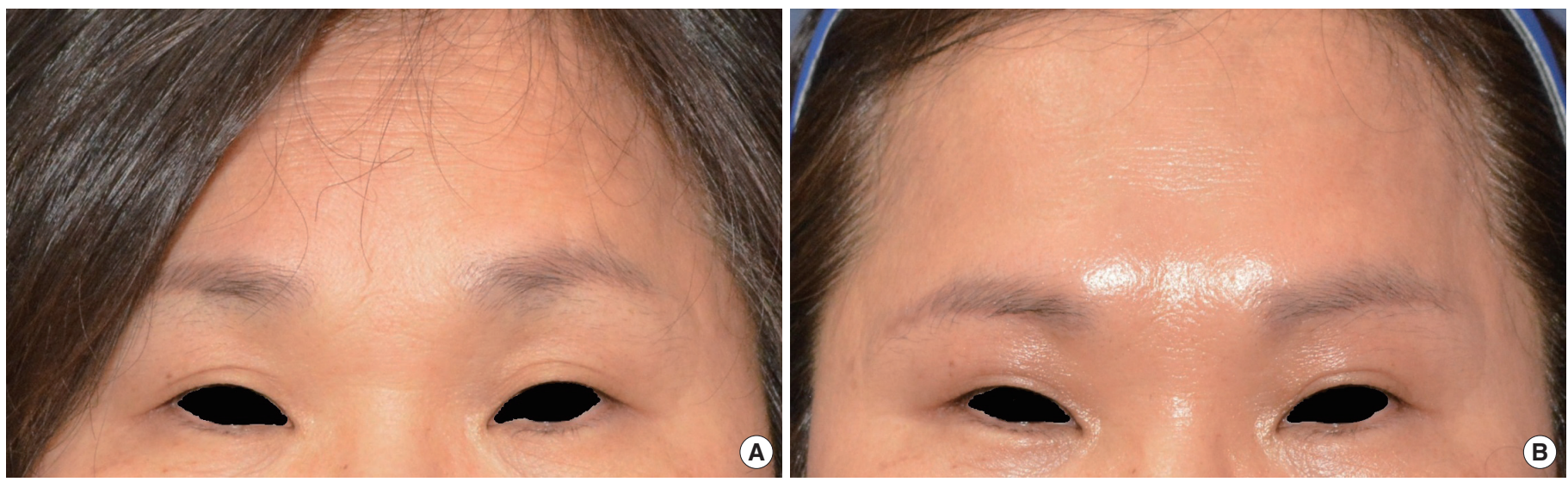

Fig. 2. Photographs of a 58-year-old female patient. Sub-brow blepharoplasty with browpexy and sunken eyelid correction were performed simultaneously. The patient wanted to correct the thick eyebrow contour, so the excision included a certain amount of the eyebrow in order to create thin eyebrows. The sunken eyelid was corrected while maintaining the shape of her orbital fissure. Improvement of brow ptosis and lateral hooding reduced the excessive compensation of the frontalis muscle and improved the forehead wrinkles. (A) Preoperative photograph and (B) photograph taken 8 months postoperatively.
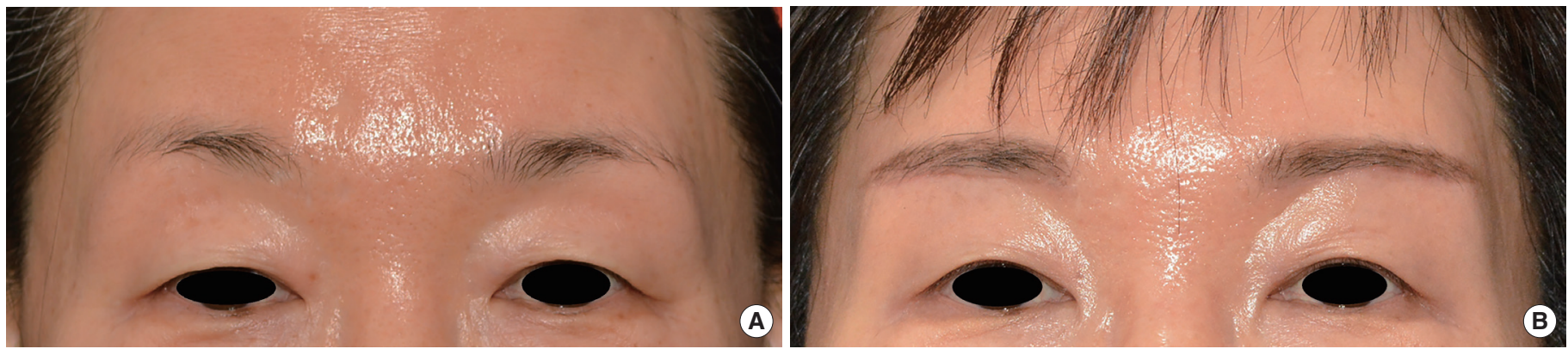

Fig. 3. Photographs of a 62-year-old female patient. The patient visited our clinic for correction of sunken eyelid and dermatochalasis with lateral hooding. Although her double fold was shallow, she did not want to make any major changes in her eyes, and was planning an eyebrow tattoo for the absent eyebrows. The same operation was performed and tattooing was performed on the eyebrow along the incision line 2 months after the operation. The sunken eyelid and lateral hooding of the upper eyelid were corrected. (A) Preoperative photograph and (B) photograph taken 6 months postoperatively. 

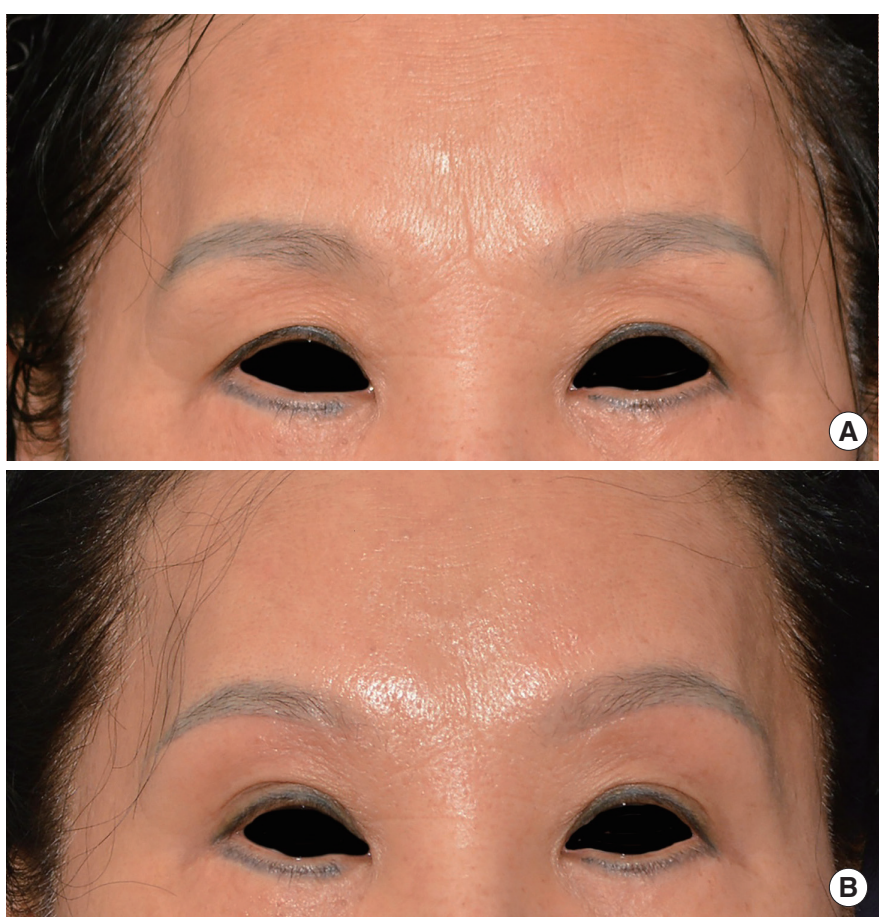

Fig. 4. Photographs of a 63-year-old female patient. The patient visited to correct the tired impression created by hooding of the upper eyelid. She had a tattooed eyebrow, did not want to change the original double fold line, and requested correction of the accompanying sunken eyelid. Making the incision along the tattooed eyebrow line allowed the scar to be concealed. (A) Preoperative photographs and (B) photograph taken 12 months postoperatively.

However, most studies showed high long-term relapse rates due to the wide range of dissection, inadequate management of brow ptosis, and insufficient fixation. Moreover, cases of sunken eyes required additional fat grafting.

With our method, adequate brow positioning can be achieved through suture fixation of the orbital portion of the upper-flap OOM to the periosteum of the supraorbital rim, while the excised hooding upper eyelid can simultaneously be buried in the lower flap to correct sunken eyelids and restore young and natural-appearing eye contours. We performed this operation on 84 patients who were suitable for this operation and were satisfied in most cases (Figs. 2-4).

During SBB, the risk of wound dehiscence, hypertrophic scarring, and trauma to the SON and/or STN must be considered. In our method, the dissection was limited to the minimum amount necessary for exposure of the periosteum, and the upper flap was fixed to the periosteum of the supraorbital rim with 3-point suturing, followed by layer-by-layer lower flap suturing for fixation to reduce tension and to minimize wound dehiscence and hypertrophic scarring. There were no cases of hypertrophic scarring or suture revision for wound dehiscence. Although some patients experienced forehead tingling and head pain during muscle splitting or numbness for several days after surgery, all recovered completely within a few months. Vertically splitting the muscle after identifying the SON and STN and performing fixation to the periosteum with no or limited dissection through windows may have been helpful.

The limitations of our method include the fact that the excised components of some areas of skin, fat, and muscle were used for sunken eyelids correction and that, because fat and muscle may atrophy or be absorbed over time, this method is not suitable for correcting severely sunken eyelids. To overcome this, we tried to improve the long-term results by elevating the excised muscle with the central portion attached to the OOM of the upper flap and then burying it under the lower flap in severely sunken eyelids. Moreover, because browpexy for appropriate brow positioning was performed using suture fixation without suprabrow excision, at the time of the operation, the eyebrow could be fixed up to a maximum of $8 \mathrm{~mm}$; if additional brow lifting was required, another surgical method, such as suprabrow excision or forehead lift, was used. Therefore, this technique is more appropriate for brow position and securing than for brow lifting. In the future, we will need a more long-lasting browpexy method that provides better securing by attempting to fix the upper-flap OOM to the frontalis muscle or deep galea located above the current fixation site by a minimally invasive method.

Although applicable in all cases of upper blepharoplasty, our method was ideal for patients with dermatochalasis and severe lateral hooding, as well as those with sunken eyelids. Satisfaction was higher in patients who were already satisfied with their current double-fold line or orbital fissure shape and in those who desired contour correction of tattooed eyebrows. Moreover, satisfaction was also high in patients who desired additional correction of sunken eyelids instead of fat grafting.

In conclusion, the method described in this study can be used to achieve periorbital rejuvenation in patients with severe dermatochalasis, lateral hooding, and sunken eyelids, while maintaining their original eye contour.

\section{CONFLICT OF INTEREST}

No potential conflict of interest relevant to this article was reported.

\section{ETHICAL APPROVAL}

The study was performed in accordance with the principles of the Declaration of Helsinki.

\section{PATIENT CONSENT}

The patients provided written informed consent for the publication and the use of their images. 


\section{ORCID}

Jin Bin Kim (https://orcid.org/0000-0001-8053-5213)

Jong Chan Kim (https://orcid.org/0000-0002-6551-0138)

Han Byeol Jin (https://orcid.org/0000-0002-0965-4372)

Sun Ok Kim (https://orcid.org/0000-0003-4783-7425)

Jee Hyeok Chung (https://orcid.org/0000-0003-2782-8898)

\section{REFERENCES}

1. Maniglia JJ, Maniglia RF, Jorge dos Santos MC, et al. Surgical treatment of the sunken upper eyelid. Arch Facial Plast Surg 2006;8:269-72.

2. Jung JM, Choi WS, Jo MS. Correction of the sunken upper eyelid depending on the dermatochalasis or blepharoptosis using the autologous fat grafting. Arch Aesthetetic Plast Surg 2014;20:148-54.

3. Collar RM, Boahene KD, Byrne PJ. Adjunctive fat grafting to the upper lid and brow. Clin Plast Surg 2013;40:191-9.

4. Park S, Kim B, Shin Y. Correction of superior sulcus deformity with orbital fat anatomic repositioning and fat graft applied to retro-orbicularis oculi fat for Asian eyelids. Aesthetic Plast Surg 2011;35:162-70.

5. Lee Y, Kwon S, Hwang K. Correction of sunken and/or multiply folded upper eyelid by fascia-fat graft. Plast Reconstr Surg 2001;107:15-9.

6. Mutou Y. Use of a silicone bag-gel prosthesis to fill in a supratarsal de- pression of the upper eyelid. Plast Reconstr Surg 1978;62:862-5.

7. Kunjur J, Sabesan T, Ilankovan V. Anthropometric analysis of eyebrows and eyelids: an inter-racial study. Br J Oral Maxillofac Surg 2006;44:8993.

8. Kim JH, Lee IJ, Park MC, et al. Aesthetic blepharoptosis correction with release of fibrous web bands between the levator aponeurosis and orbital fat. J Craniofac Surg 2012;23:e52-5.

9. Yuzuriha S, Matsuo K, Kushima H. An anatomical structure which results in puffiness of the upper eyelid and a narrow palpebral fissure in the Mongoloid eye. Br J Plast Surg 2000;53:466-72.

10. Lee D, Law V. Subbrow blepharoplasty for upper eyelid rejuvenation in Asians. Aesthet Surg J 2009;29:284-8.

11. Parkes ML, Kamer FM, Merrin ML. Infrabrow lift. Laryngoscope 1976; 86:1869-72.

12. Kim YS, Roh TS, Yoo WM, et al. Infrabrow excision blepharoplasty: applications and outcomes in upper blepharoplasty in Asian women. Plast Reconstr Surg 2008;122:1199-205.

13. Ichinose A, Sugimoto T, Sugimoto I, et al. Extended infrabrow excision blepharoplasty for dermatochalasis in Asians. Arch Facial Plast Surg 2011;13:327-31.

14. Kim YS. Subbrow blepharoplasty using supraorbital rim periosteal fixation. Aesthetic Plast Surg 2014;38:27-31. 